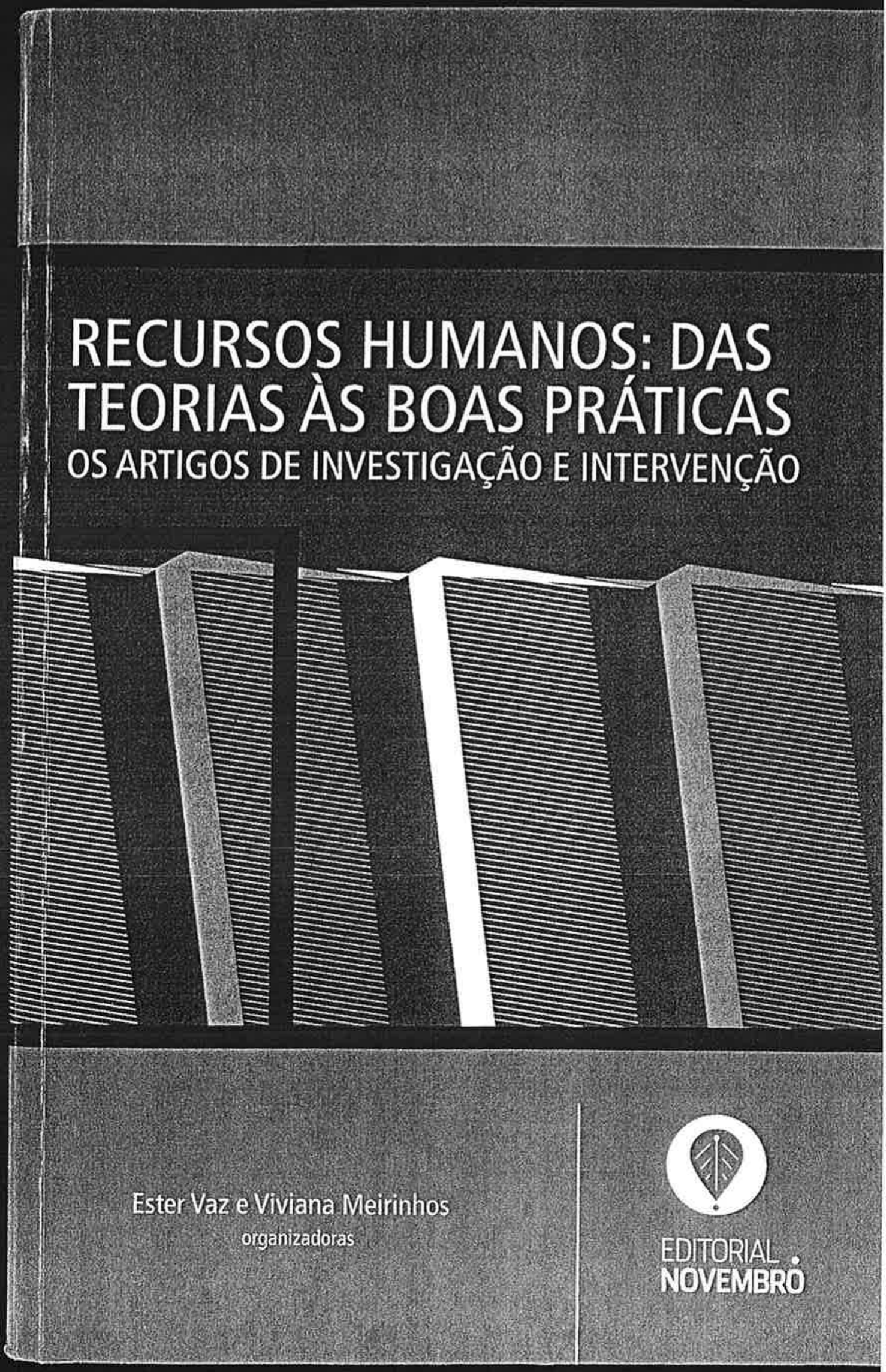





\section{OS SISTEMAS DE INFORMAÇÃO E AS PRÁTICAS DE RECURSOS HUMANOS NAS ORGANIZAÇÕES DA REGIÃO DE SETÚBAL}

JOSÉ MANUEL GAMEIRO REBELO SANTOS

jrebelo@esce.ips.pt

MARIA DE LURDES CARDINA PEDRO

mlpedro@pep.pioneer.pt

JOÃO PEDRO CORDEIRO

jcordeiro@esce.jps.pt 


\section{RESUMO}

Os Sistemas e as Tecnologias de Informação vêm assumindo um lugar de cada vez mais importância para as práticas organizacionais em geral e para as práticas da Gestão de Recursos Humanos em particular.

Atendendo a que frequentemente não se trata de sistemas integrados perdemse sinergias. A questão que se coloca é se isso se deve ao desconhecimento dos produtos existentes no mercado e das suas potencialidades e/ou à impreparação técnica dos profissionais de Gestão de Recursos Humanos.

Com esta comunicação propomo-nos apresentar dados preliminares relativos a: identificação e caracterização das principais Tecnologias de Informação existentes no mercado para a Gestão de Recursos Humanos; identificação das Tecnologias de Informação mais utilizadas nas organizações da região e as razões da escolha; e caracterização das opções privilegiadas pelas organizações no âmbito da utilização.

Os resultados esperados centram-se no conhecimento do software disponível para Gestão de Recursos Humanos, bem como nas suas características e potencialidades, o que constitui um dos aspectos a concretizar de inegável mais-valia. Um outro resultado reporta à percepção relativa às Tecnologias de Informação utilizadas para gerir Recursos Humanos, bem como à forma como estas são utilizadas permitindo uma melhor compreensão daquilo que é actualmente valorizado pelas organizações com actividade na região de Setúbal.

\section{INTRODUÇÃO}

Vivemos na era da informação, na qual se exige das organizações a utilização de ferramentas e instrumentos que potencializem a recolha e tratamento da informação com celeridade contribuindo para a tomada de decisões.

Os Recursos Humanos são o activo mais importante das organizações pelo que se justifica o recurso a sistemas e tecnologias que possibilitem a monitorização e o desenvolvimento destes activos tão valiosos. É nesse sentido que surge esta investigação: conhecer os sistemas e tecnologias utilizados pelas organizações da região de Setúbal para a Gestão de Recursos Humanos. Pretende-se mesmo ir um pouco mais longe e compreender melhor a forma como as organizações utilizam estes sistemas e tecnologias, ou seja, são utilizados para quê? 
São, pois, estes os objectivos do estudo que se alicerça na revisão da literatura e em recolha de informação através de inquérito por questionário a organizações da região de Setúbal.

Saliente-se que esta comunicação reporta apenas a apresentação de dados preliminares relativos a um projecto mais vasto delineado para um período de 24 meses, pelo que os dados apresentados reportam a uma análise exploratória com base a inquéritos por entrevista a peritos, a testemunhas privilegiadas com um bom conhecimento da temática.

\section{O PAPEL DOS SISTEMAS E DAS TECNOLOGIAS DE INFORMAÇÃO NAS PRÁTICAS ORGANIZACIONAIS DE GESTÃO DE RECURSOS HUMANOS}

Embora prolifere uma vasta bibliografia sobre Sistemas e Tecnologias de Informação, verificando-se também a existência de um número substancial de obras que articulam os SI e as TI com a Gestão de Recursos Humanos, $o$ facto é que trabalhos de natureza empírica sobre o tema são em número muito diminuto. Se nos circunscrevermos à região de Setúbal são mesmo raros.

As Tecnologias de Informação têm assumido, de qualquer forma, um peso crescente e um papel determinante tanto no funcionamento das organizações, como da própria economia (Carvalho e Gaspar, 2001).

Esta situação tem potenciado o desenvolvimento de novas formas de organização do trabalho, nomeadamente o tele-trabalho (Rebelo, 1999) e suscitado uma procura crescente de trabalhadores com qualificações neste domínio e a emergência de uma multiplicidade de aplicações direccionadas para as organizações (Carvalho e Gaspar, 2001), algumas das quais para a Gestão de Recursos Humanos.

Também a emergência de empresas virtuais constitui um novo desafio quer para os Sistemas de Informação e as Tecnologias de Informação, quer para a própria Gestão de Recursos Humanos, exigindo, nomeadamente redefinição de processos e de procedimentos (Azevedo, 2000).

A proliferação e desenvolvimento de Sistemas e Tecnologias de Informação para gerir os Recursos Humanos está a possibilitar que algumas actividades e inerentes tarefas anteriormente da exclusiva responsabilidade dos Gestores e Técnicos de Recursos Humanos passem a ser realizadas de forma automática (pelo menos em parte) ou por outros gestores, ou ainda pelos próprios trabalhadores, a quem as informações a inserir, alterar ou eliminar dizem respeito (Boateng, 2007).

Como exemplo ilustrativo refira-se a possibilidade existente em muitas organizações dos trabalhadores poderem justificar as suas próprias faltas e definir e ou alterar o período de férias ou a simples consulta dos seus próprios dados referentes a férias, faltas, trabalho suplementar realizado, componentes da remuneração, situação perante a fiscalidade, retenções na fonte efectuadas no âmbito 
do Imposto sobre o Rendimento das Pessoas Singulares e até dados dos seus documentos de identificação.

A possibilidade da realização automática de certas actividades da Gestão de Recursos Humanos tem gerado alguma apreensão, sobretudo nas situações em que cabia ao Gestor de Recursos Humanos a tomada de determinadas decisões que, no limite, passaram a estar a cargo de Sistemas e Tecnologias de Informação.

Embora a grande revolução nas organizações, decorrente da utilização das Tecnologias de Informação, se tenha iniciado com o aparecimento dos computadores pessoais em fins da década de setenta e princípios da década de oitenta (Lopes, 1997), as bases de dados relacionais em rede e o tele-processamento constituíram também factores decisivos na alteração das formas de comunicação nas organizações, permitindo a partilha e a troca de informações.

O Correio Electrónico, a tele-conferência e outros instrumentos de comunicação, grande parte dos quais online, alteraram profundamente as formas de comunicar tanto a nível intra organizacional como a nível inter-organizacional (Rebelo, 1999).

Muitos destes instrumentos são hoje utilizados a nível da comunicação organizacional e da Gestão de Recursos Humanos.

O uso dos Sistemas e Tecnologias de Informação e das Tecnologias de Informação em contexto organizacional tem incrementado a eficiência tanto a nível da gestão dos seus negócios como da gestão da própria organização e da implementação dos seus objectivos estratégicos (Hussain e Prowse, 2001).

Os dados empíricos resultantes de estudos internacionais evidenciam o papel da Gestão de Recursos Humanos no desenvolvimento dos Sistemas de Informação para a Gestão de Recursos Humanos e para a gestão em geral (Hussain e Prowse, 2001).

Um estudo sobre a Gestão de Recursos Humanos no sector segurador em Portugal (Brandão, 2000) revela a emergência de funções de construção/gestão de Sistemas de Informação a nível da função Recursos Humanos. Não obstante, verifica-se que a função se encontra pouco formalizada ao nível das actividades mais recentes de Gestão de Recursos Humanos, concretamente no que respeita a construção e Gestão de Sistemas de Informação (Brandão, 2000).

Este aspecto pode dever-se ao défice de trabalhadores com competências neste domínio que já em 2000 era superior a treze mil indivíduos (Carvalho e Gaspar, 2001) e que será decerto bastante mais substancial quando se pretenda encontrar trabalhadores que conciliem competências nos domínios das Tecnologias de Informação e Comunicação e da Gestão de Recursos Humanos.

Com efeito, frequentemente identificam-se bons profissionais no âmbito da informática e também bons profissionais no domínio da Gestão de Recursos Humanos; raramente se encontram profissionais com as competências requeridas nas duas valências que referimos (SI/TI e GRH).

É face a tudo isto e tendo em conta a insuficiente produção científica que concilie as áreas dos Sistemas e tecnologias de Informação com a Gestão de Recursos humanos que nos propusemos a realizar este projecto que poderá constituir um ponto de partida para um estudo mais abrangente e multidisciplinar envolvendo investigadores das áreas de saber referidas. 
No âmbito da Gestão de Recursos Humanos os Sistemas e Tecnologias de Informação são utilizados nomeadamente no controlo de ausências/presenças, na elaboração dos ficheiros de cadastro de pessoal, no processo de recrutamento e selecção, no processamento das remunerações, no planeamento de Recursos Humanos, na Formação Profissional, na Avaliação de Desempenho e na auditoria e controlo (Balanço social, tableau de bord) (Rascão 2001), conforme consta no quadro seguinte.

QUADRO 1. PRINCIPAIS ACTIVIDADES DE GRH EM QUE SE RECORRE A SI E TI

\begin{tabular}{|c|c|}
\hline \multirow{4}{*}{$\begin{array}{c}\text { UTILIZAÇĀO DE SISTEMAS DE INFORMAÇĀ0 E } \\
\text { TECNOLOGIAS DE INFORMAÇÃO NA GESTÃO DE } \\
\text { RECURSOS HUMANOS }\end{array}$} & ACTIVIDADES \\
\cline { 2 - 2 } & Controlo do absentismo (ausências/presenças) \\
\cline { 2 - 2 } & Ficheiros de cadastro do Pessoal \\
\cline { 2 - 2 } & Recrutamento e Selecção \\
\cline { 2 - 2 } & Formação Profissional \\
\cline { 2 - 2 } & Avaliaçāo de Desempenho \\
\cline { 2 - 2 } & Auditoria e Controlo \\
\hline
\end{tabular}

Nalgumas organizações, as actividades de Gestão de Recursos Humanos em que se utilizam Sistemas e Tecnologias de Informação não se reduzem às constantes no quadro anterior. De qualquer forma, são estas as principais utilizações conforme corroboram os dados preliminares que apresentamos de seguida.

\section{APRESENTAÇÃO DE ALGUNS RESULTADOS PRELIMINARES}

De acordo com informações preliminares obtidas em fase exploratória junto de peritos e testemunhas privilegiadas, a utilização dos Sistemas e Tecnologias de Informação na Gestão de Recursos Humanos nas organizações pode ser entendido no quadro de duas realidades distintas:

- Organizações em que se verifica a utilização integrada de Sistemas e Tecnologias de Informação no âmbito duma gestão estratégica e de desenvolvimento dos Recursos Humanos; possuem sistemas complexos e recorrem a tecnologias sofisticadas e de elevado preço;

- Organizações em que se verifica uma utilização incipiente das novas tecnologias, sendo o seu uso restringido à gestão administrativa de Recursos Humanos, concretamente ao processamento salarial, à gestão e controlo do tempo de trabalho e à resposta às exigências decorrentes do cumprimento das obrigações legais; possuem sistemas 
muito simples (por vezes chegam a ser rudimentares) e recorrem a tecnologias básicas de baixo preço;

- Uma eventual terceira realidade, situada algures entre a primeira e a segunda e em que as Tecnologias de Informação são utilizadas na Gestão Administrativa de Recursos Humanos e também a Gestão da Formação e nalguns casos na Gestão do Sistema de Saúde, Segurança e Higiene no Trabalho. Trata-se, portanto, de uma realidade intermédia e, consequentemente, possuem sistemas simples, mas não rudimentares, e recorrem a tecnologias já de alguma sofisticação com preços médios.

Embora não pretendamos construir uma tipologia das organizações que indubitavelmente associe à primeira realidade as organizações tipo " $X$ ", à segunda realidade as organizações tipo "Y" e à terceira realidade as organizações tipo "Z", o facto é que tendencialmente são as organizações que adoptam práticas de gestão avançada (normalmente multinacionais, empresas nacionais de maior dimensão ou pelo menos com notoriedade) aquelas que utilizam de forma integrada sistemas e Tecnologias de Informação, visando uma gestão integrada de Recursos Humanos, no âmbito de um planeamento estratégico.

Já em relação à utilização restrita de istemas e Tecnologias de Informação, constata-se que a sua ocorrência, preponderantemente, verifica-se em sectores de actividade menos desenvolvidos ou que tradicionalmente não necessitam de muita tecnologia.

No que reporta a terceira realidade, associamo-Ia a organizações e empresas de média dimensão e a grande parte das organizações que integram a administração pública, bem como o sector cooperativo e as Instituições Particulares de Solidariedade Social.

Neste âmbito, como referiu um dos entrevistados, "variáveis como a origem do capital social (nacional ou estrangeiro), o sector de actividade, tipo de gestão (pública ou privada), a formação académica dos gestores, entre outros, poderão eventualmente assumir um papel impulsionador ou redutor na implementação e utilização de sistemas e tecnologias de informação integrados no negócio e na estratégia da organização".

Um aspecto digno de registo é o da coexistência de vários programas informáticos para gerir Recursos Humanos nalgumas organizações mesmo quando pertencentes à primeira realidade. Mais curioso ainda é quando coexistem vários programas, entre os quais um de maior potencial que por si só poderia responder a todas as necessidades da organização tanto em Gestão de Recursos Humanos como em Gestão em geral. Esta constatação reforça a nossa percepção relativa à dificuldade em conciliar elevadas competências de Informática/Sistemas de Informação/Tecnologias de Informação e Gestão de Recursos Humanos. 


\section{SÍNTESE CONCLUSIVA}

Apesar do desenvolvimento sem precedentes que as Tecnologias e os Sistemas de Informação conheceram nas últimas décadas, não obstante os imperativos legais levarem à sua disseminação quer individualmente quer organizacionalmente, o facto é que nesse domínio se está ainda bastante aquém do desejável, verificando-se que em muitas organizações a iliteracia informática ainda é uma realidade.

A nível organizacional e no âmbito da Gestão de Recursos Humanos constata-se que muito frequentemente a utilização dos meios informáticos, que muitas vezes até já existem na organização, se restringe ao mínimo necessário para cumprir as obrigações legais.

\section{BIBLIOGRAFIA}

Azevedo, Américo L. (2000). A emergência da empresa virtual e os requisitos para os sistemas de informação, in Gestão e Produção, Volume 7, n. ${ }^{\circ}$, Porto, Faculdade de Engenharia da universidade do Porto, pp. 208-225.

Boateng, Asafo-Adjei Agyenim (2007). The Role of Human Resource Information Systems (HRIS), in Strategic Human Resource Management (SHRM), Master of Science Theses in Accounting, Swedish School Of Economics and Business Administration.

Brandão, Ana Maria (2000). Modelos de Gestão dos Recursos Humanos - o caso do sector segurador em Portugal, in Sociedade e Cultura 1, Cadernos do Noroeste, Série sociologia, Volume 13, n. ${ }^{\circ} 1$, Braga, CCHS, Universidade do Minho, pp. 181-200.

Carvalho, Paulo Soeiro, Gaspar, Teresa Dias (2001). Mão-de-obra digital: o bem mais escasso da nova economia, in Prospectiva e Planeamento, Volume 7, Lisboa, DPP, pp. 295-351.

Hussain, Zahid, Prowse, Peter (2004). Human Resource Information Systems (HRIS) as Means of Fulfilling Job Roles More Professionally for Human Resource (HR) Managers, Working paper n. ${ }^{\circ} 04 / 07$, Bradford University School of Management.

Lopes, Maria Joana de Menezes (1997). Sistemas de Informação para a Gestão, Lisboa: Universidade Aberta.

Rascão, José (2001). Sistemas de Informação para as Organizações, Lisboa, Edições Sílabo. 
Rebelo. José, (1999). Efeitos perversos das Tecnologias de Informação na Cultura Organizacional, in Machado, C. G., Melo, M., Franco, V., Santos, N.,(org) (2000), Interfaces da Psicologia, Vol 2, Évora, Departamento de Pedagogia e Educação da Universidade de Évora, pp. 355-364. 


\section{ESTRATÉGIAS EMPRESARIAIS E GESTÃO DE RECURSOS HUMANOS: ESTUDOS DE CASO NO SECTOR DE COMPONENTES PARA AUTOMÓVEL}

JOÃO PEDRO CORDEIRO

ESCOLA SUPERIOR DE CIÊNCIAS EMPRESARIAIS / IPS

jcordeiro@esce.ips.pt 


\section{RESUMO}

Os directores/gestores das empresas encontram, ainda hoje, dificuldade em estabelecer ligações entre as práticas de Gestão de Recursos Humanos e a estratégia empresarial. Todavia, é inquestionável que as empresas com pessoal qualificado e comprometido (committment) possuem maior capacidade para implementar as estratégias concebidas. Deve, pois, haver a preocupação de colocar nas empresas profissionais altamente qualificados e competentes, que serão os principias responsáveis pela identificação e implementação das estratégias. Assim sendo, esta comunicação, inserida numa investigação de doutoramento, pretende reflectir sobre as inter-relações entre as estratégias empresariais e as práticas de Gestão de Recursos Humanos. O objecto de estudo é os quadros superiores das empresas do sector de componentes para automóvel. A metodologia utilizada foi o estudo de caso em três empresas do mesmo sector. Os resultados sugerem que as empresas do sector de componentes para automóvel, ao possuírem diferentes estratégias empresariais, possuem também diferentes formas de preconizar essas mesmas estratégias, as quais sustentam e são sustentadas por diferentes práticas de Gestão de Recursos Humanos, num processo de (retro) alimentação mútua. A abordagem vai, assim, na direcção de uma concepção da Gestão de Recursos Humanos entendida como um "processo" que influencia e é influenciado pela especificidade dos contextos organizacionais, traduzidos pelas diferentes estratégias empresariais adoptadas.

\section{INTRODUÇÃO}

O reconhecimento da importância do conhecimento como fonte de vantagem competitiva traz consequências óbvias para a Gestão de Recursos Humanos já que determina que as políticas e as práticas de Gestão de Recursos Humanos sejam revistas e integradas num sistema coerente e consistente. As estratégias empresariais devem influenciar e ser influenciadas pelas estratégias de Recursos Humanos, num processo de retro-alimentação.

Tendo em consideração que o tema central desta comunicação é "Estratégias Empresariais e Gestão de Recursos Humanos", podemos formular o problema central desta comunicação pela seguinte questão: de que forma as práticas de gestão dos quadros se relacionam com as estratégias nas empresas do sector de componentes para automóvel? 